OPEN

SUBJECT AREAS:

X-RAY

CRYSTALLOGRAPHY

BIOLOGICAL PHYSICS

Received

20 March 2014

Accepted

30 June 2014

Published

21 July 2014

Correspondence and requests for materials should be addressed to

R.M. (ryuta@tokai-u.jp)

\title{
Spatiotemporal development of soaked protein crystal
}

\author{
Ryuta Mizutani' ', Yusuke Shimizu', Rino Saiga' ', Go Ueno², Yuki Nakamura ${ }^{3}$, Akihisa Takeuchi ${ }^{4}$, \\ Kentaro Uesugi ${ }^{4} \&$ Yoshio Suzuki ${ }^{4}$
}

'Department of Applied Biochemistry, School of Engineering, Tokai University, Kitakaname 4-1-1, Hiratsuka, Kanagawa 2591292, Japan, ${ }^{2}$ RIKEN SPring-8 Center, Kouto 1-1-1, Sayo, Hyogo 679-5148, Japan, ${ }^{3}$ Rigaku Corporation, 14-8, Akaoji-cho, Takatsuki-Shi, Osaka 569-1 146, Japan, ${ }^{4}$ Research and Utilization Division, JASRI/SPring-8, Kouto 1-1-1, Sayo, Hyogo 679-5 198, Japan.

Crystal soaking is widely performed in biological crystallography. This paper reports time-resolved X-ray crystallographic and microtomographic analyses of tetragonal crystals of chicken egg-white lysozyme soaked in mother liquor containing potassium hexachloroplatinate. The microtomographic analysis showed that X-ray attenuation spread from the superficial layer of the crystal and then to the crystal core. The crystallographic analyses indicated that platinum sites can be classified into two groups from the temporal development of the electron densities. A soaking process consisting of binding-rate-driven and equilibrium-driven layers is proposed to describe these results. This study suggests that the composition of chemical and structural species resulting from the soaking process varies depending on the position in the crystal.

C rystal soaking is widely performed in biological crystallography. Small ligand molecules can be introduced into a native crystal in order to obtain a protein-ligand complex. Heavy atom derivatives are prepared by soaking native crystals in mother liquor containing heavy atom reagents. Crystal soaking is also performed to adjust the mother liquor conditions, such as $\mathrm{pH}$ and precipitant concentration, prior to crystallographic data collection.

Soaked protein crystals have been visualized by light microscopy. In particular, lysozyme crystals can be studied by fluorescence microscopy by infusing them with fluorescent surfactants ${ }^{1}$. The anisotropic diffusion of fluorescein into lysozyme crystals has been analyzed by confocal microscopy ${ }^{2}$. A simulation of small molecules diffusing into protein crystals has been reported ${ }^{3}$. The time-dependence of platinum binding to lysozyme has been studied by protein crystallography $y^{4,5}$. However, the relationship between the three-dimensional distribution of chemical compounds in crystals and their coordination to protein has not been delineated.

This paper reports X-ray crystallographic and microtomographic studies of tetragonal crystals of chicken eggwhite lysozyme soaked in mother liquor containing potassium hexachloroplatinate. A time-resolved threedimensional visualization of the soaking process was performed by synchrotron radiation microtomography. The platinum first entered the superficial layer of the crystal and gradually penetrated to the crystal core. The three-dimensional structure of the platinum derivative in each soaking period was determined by time-resolved crystallography. These analyses revealed the spatial distribution of platinum and its correlation with crystal structures.

\section{Results}

Heavy atom distribution in crystal. The three-dimensional distribution of X-ray linear attenuation coefficients (LACs) of the sample was reconstructed from each microtomographic dataset taken during the soaking process. Supplementary Figure S1 shows a rendering of a soaked crystal attached to the capillary inner wall. The LAC distributions along the midline perpendicular to the ( 110$)$ face of the crystal are shown in Fig. 1. Overshoots and undershoots were observed at interfaces between material phases, such as mother liquor and crystal. These overand undershoots were ascribed to a refraction effect caused by the microtomography setup, in which the detector was placed $20 \mathrm{~mm}$ away from the crystal to make space for sample rotation. Besides these effects, the LAC of the crystal increased during the soaking process, while the LAC of surrounding mother liquor was almost constant. Figure $2 \mathrm{a}$ shows virtual cross sections of the crystal during soaking. These cross sections indicated that X-ray attenuation spread from the superficial layer and then to the crystal core. 


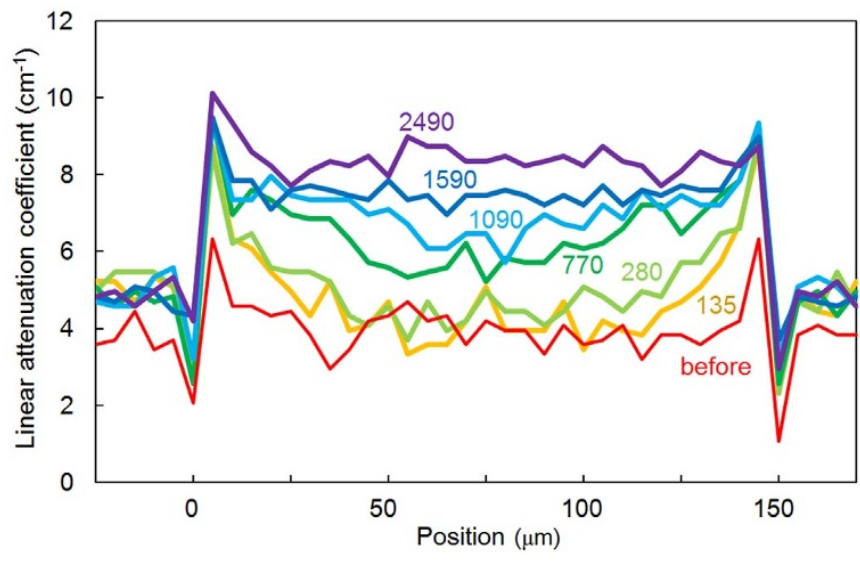

Figure $1 \mid$ LAC distributions along the midline perpendicular to the (1 11 0 ) face. Each distribution is differentiated by color and labeled with soaking time (s).

Before soaking, the average LAC of the crystal above the Pt $\mathrm{L}_{\mathrm{III}}$ edge $(11.580 \mathrm{keV})$ was $4.2 \mathrm{~cm}^{-1}$ (Table 1$)$, which is the same as that below the edge $(11.550 \mathrm{keV})$. After soaking for $2490 \mathrm{~s}$, the average LAC at $11.580 \mathrm{keV}\left(8.6 \mathrm{~cm}^{-1}\right)$ was obviously higher than that at
$11.550 \mathrm{keV}\left(6.7 \mathrm{~cm}^{-1}\right)$. These results indicate that the LAC increase is ascribable to platinum ions. The LAC at $11.580 \mathrm{keV}$ after soaking for $2490 \mathrm{~s}$ is equivalent to the incorporation of 1.6 platinum atoms per asymmetric unit. The LAC of the surrounding mother liquor containing platinum ions was $5.0 \pm 0.5 \mathrm{~cm}^{-1}$ at $11.580 \mathrm{keV}$, which coincides with the theoretical LAC of $4.9 \mathrm{~cm}^{-1}$. The LAC of the mother liquor around the crystal did not show any apparent changes during the soak (Fig. 1). The mother liquor volume within $5 \mathrm{~mm}$ of the crystal was more than $10^{3}$ times that of the crystal volume. The platinum concentration of the mother liquor can therefore be considered constant.

Figure 3 shows the time dependence of the average LAC over the crystal volume. The LAC increased for more than $40 \mathrm{~min}$. A logarithm plot of the difference in this value from its saturation value (Fig. 3b) indicated that the time dependence after $750 \mathrm{~s}$ can be approximated with a kinetic constant $k_{1}=0.00055 \mathrm{~s}^{-1}$. The LAC of a crystal soaked for $44 \mathrm{~h}$ (Table 1 ) was used as the saturated LAC in this plot. Deviations from linearity were observed before $300 \mathrm{~s}$, indicating that the initial process should be described with another kinetic model.

It has been reported that the diffusion of surfactants into lysozyme crystals is composed of two temporal processes: a transient period of initial infusion and a subsequent saturation process on an even

a

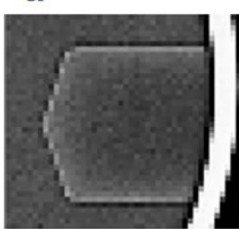

$135 \mathrm{~s}$

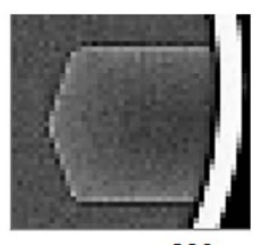

$280 \mathrm{~s}$

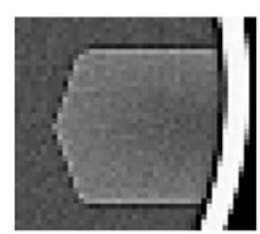

$770 \mathrm{~s}$
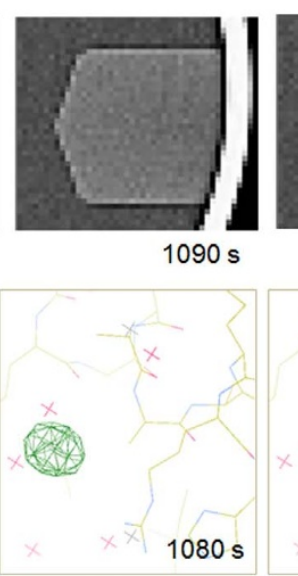

$750 \mathrm{~s}$

250 s

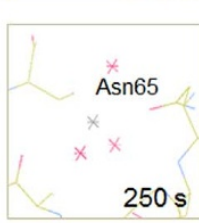

250 s
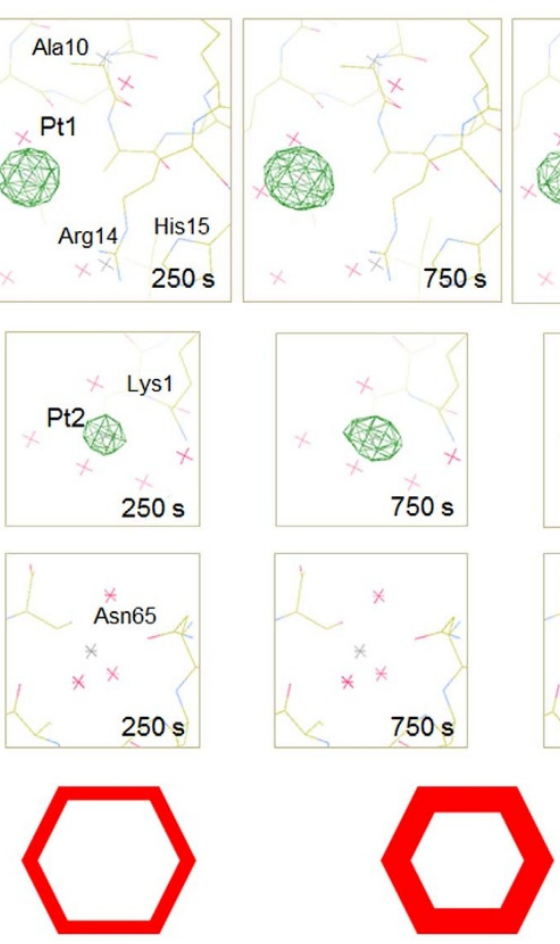

$1090 \mathrm{~s}$
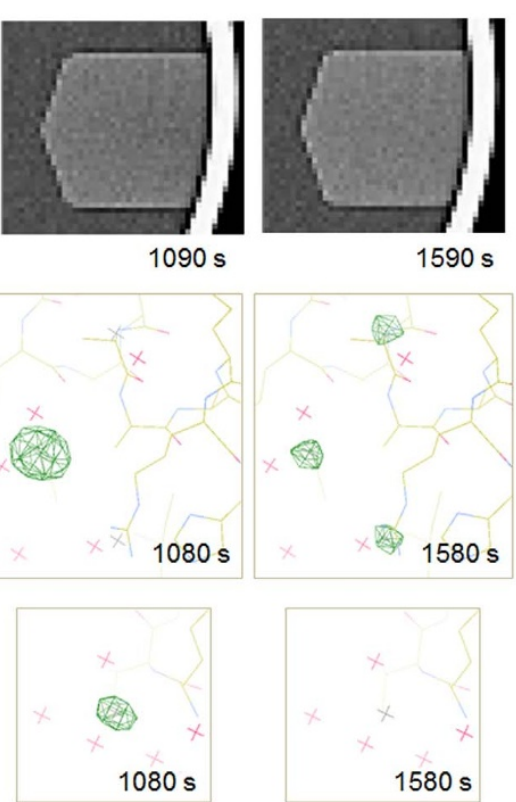

$1590 \mathrm{~s}$
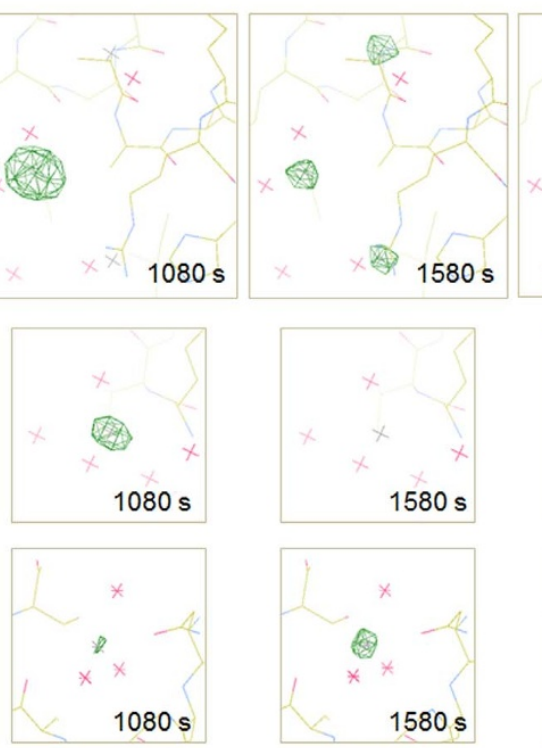

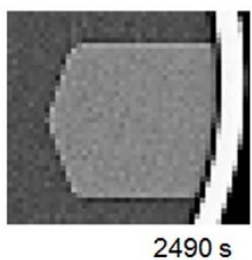

$2490 \mathrm{~s}$
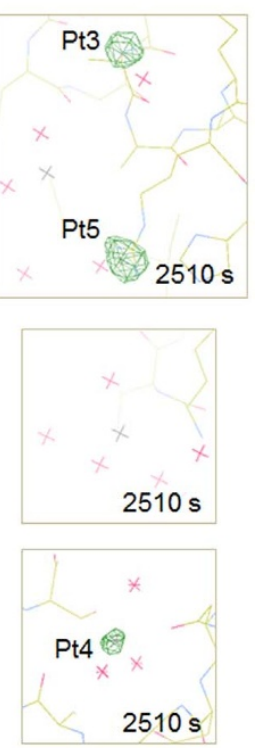

C
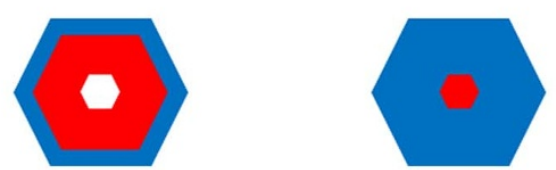

Figure $2 \mid$ Spatiotemporal development of platinum coordination in soaked crystal. (a) Microtomographic cross sections parallel to the ( 110$)$ face. Soaking times are indicated with labels. LACs are shown in gray scale from $0 \mathrm{~cm}^{-1}$ (black) to $16 \mathrm{~cm}^{-1}$ (white). (b) Electron density maps of platinum sites. Omit maps were calculated using the native model and derivative datasets, and they were contoured at 0.8 electron $\AA^{-3}$. Platinum sites and soaking times are indicated with labels. (c) Schematic representation of layered process of crystal soaking. Crystal soaking proceeds from left to right. The soaking process starts with initial permeation (red) of platinum ions into native crystal (white). The initial layer corresponds to platinum sites 1 and 2. Then the initial layer moves toward the center of the crystal and is simultaneously replaced with the succeeding layer (blue) in which platinum ions have moved to sites 3, 4, and 5. Finally, the initial layer reaches the crystal center and the succeeding layer dominates the entire crystal. 
Table 1 | Time dependence of crystal LAC

\begin{tabular}{lcccccccc} 
Soaking $(\mathrm{s})$ & Native & 135 & 280 & 770 & 1090 & 1590 & 2490 & $44 \mathrm{~h}$ \\
\hline LAC at $11.580 \mathrm{keV}\left(\mathrm{cm}^{-1}\right)^{\mathrm{a}}$ & $4.2 \pm 0.5$ & $5.6 \pm 1.3$ & $6.1 \pm 1.3$ & $7.1 \pm 1.0$ & $7.5 \pm 0.9$ & $7.9 \pm 0.8$ & $8.6 \pm 0.9$ & $9.5 \pm 0.5$ \\
LAC at $11.550 \mathrm{keV}\left(\mathrm{cm}^{-1}\right)^{\mathrm{a}}$ & $4.2 \pm 0.4$ & 0.5 & 0.7 & 1.1 & 1.2 & 1.4 & 1.6 & 2.0 \\
Equivalent platinum ions per asymmetric unit & - & 0.5 & & & & & &
\end{tabular}

longer time scale ${ }^{1}$. Those authors discussed their observation that the concentration difference between the edge and core of the crystal in the initial process was virtually independent of mass transfer outside the crystal and instead controlled by diffusion in the crystal. Since platinum ions specifically bind to lysozyme molecules, platinum diffusion in the crystal depends on its binding rate. The initial increase in LAC before $300 \mathrm{~s}$ results from such a process. On the other hand, the subsequent process represented by the constant $k_{1}$ should be affected by transport from the surrounding solution. Calculation models have been proposed for evaluating these soaking processes by introducing physical and chemical parameters ${ }^{2,3}$. However, it is difficult to describe the protein-ligand interaction using only kinetic constants. The crystallographic analysis of each soaking period described below revealed the processes underlying these observations.

Heavy atom coordination in crystal. Since the microtomographic analysis indicated that the spatiotemporal heterogeneity was caused

\section{a}

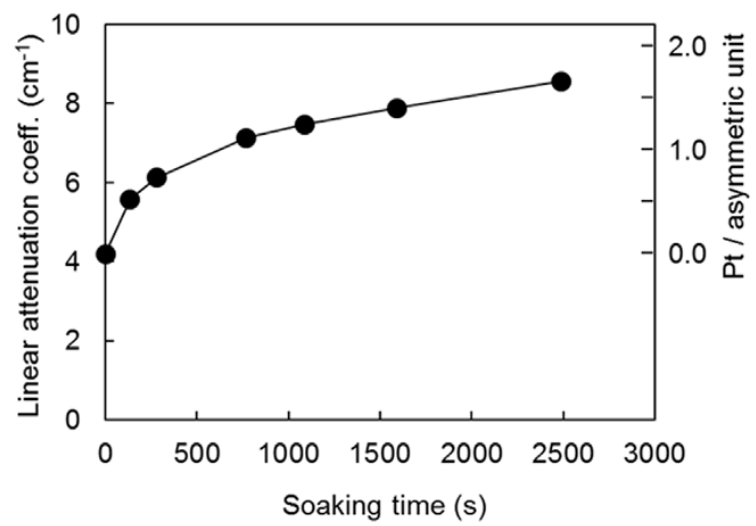

b

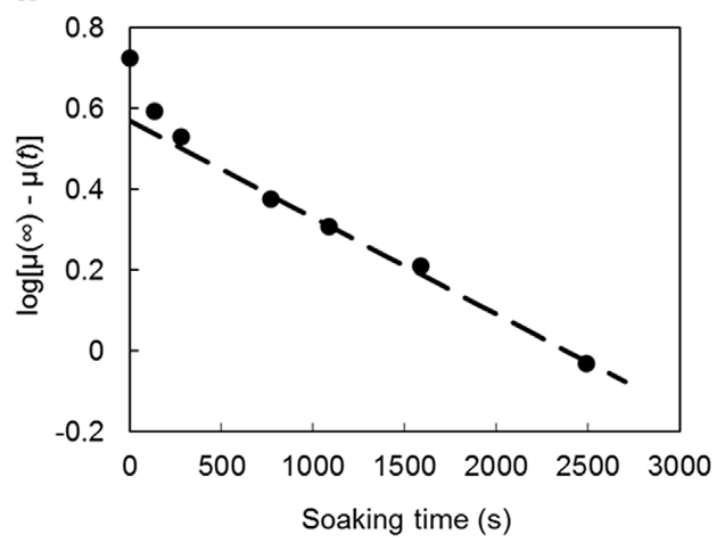

Figure $3 \mid$ (a) Time dependence of average LAC over the crystal volume. The equivalent number of platinum ions in the asymmetric unit is indicated on the right axis. (b) Logarithm plot of the LAC difference from saturation. The time dependence after $750 \mathrm{~s}$ can be approximated with a kinetic constant $0.00055 \mathrm{~s}^{-1}$, which is represented by a broken line. by soaking, the soaked crystal was subjected to time-resolved crystallography. Time-series omit maps were calculated using the native model and derivative datasets (Fig. 2b) collected from one crystal of $120-130 \mu \mathrm{m}$ in each dimension. Platinum coordination sites with electron densities higher than 1.0 electron $\AA^{-3}$ were found at five positions, as summarized in Table 2. These sites corresponded to the peaks in the Bijvoet difference maps (Supplementary Fig. S2) taken at $750 \mathrm{~s}$ and $3610 \mathrm{~s}$, and this indicates that platinum ions existed at these sites. Another density at the position of water 180 of the starting model $2 \mathrm{LYM}^{6}$, which was removed owing to close contact with water 131, was also observed. This density was used as a reference peak in the omit maps, and it was almost constant throughout the soaking process (Table 2). The dose accumulated throughout the period of the crystallographic data collection was estimated from the microtomographic LAC to be 1.1-1.3 $\times 10^{5} \mathrm{~Gy}$, which is less than $1 \%$ of the Henderson limit $(2$ $\left.\times 10^{7} \mathrm{~Gy}\right)^{7}$. Note that we took other datasets with a pretreatment dose of approximately $0.5 \times 10^{5}$ Gy before crystallographic data collection, but there were no major differences from those without the pretreatment dose in regard to the development of platinum sites.

Figure 4 shows the time dependence of the electron densities at the platinum sites. These sites can be classified into two groups on the basis of the time dependence of electron density development. The first group is composed of sites 1 and 2. Electron densities of these sites were less than 0.2 electron $\AA^{-3}$ in the native structure. They rapidly reached maximum at $750 \mathrm{~s}$ and then gradually decreased until $2510 \mathrm{~s}$. These platinum sites were in interaction with water molecules, but were located more than $4 \AA$ away from the protein. We suggest that these sites were not occupied by other ions or molecules in the native crystal, and hence, they easily accommodated platinum ions. These sites coincide with the platinum sites reported for crystals soaked for $10 \mathrm{~min}^{5}$.

In contrast to labile sites 1 and 2 , another group of platinum sites3, 4, and 5-became dominant after soaking for $1500 \mathrm{~s}$. Since the distance between sites 1 and 5 was $5.4 \AA$ and the $\mathrm{Pt}-\mathrm{Cl}$ bond length ${ }^{8}$ is $2.3 \AA$, the coordination of site 1 was interchanged with that of site 5 . Sites 3 and 4 are close $(<2 \AA)$ to the positions of water 153 and 141 of the native model. Site 3 is located 3.0 A from Leu 129 OXT, although the structure of the Leu 129 carboxyl group was poorly determined owing to its low electron density. Site 4 is located $2.5 \AA$ from Asn 65 O $\delta 1$. Site 5 is located $2.4 \AA$ from His $15 \mathrm{C} \varepsilon 1$ and $2.8 \AA$ from Arg 14

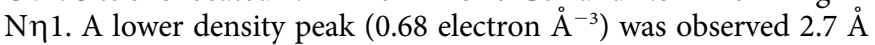
from His $15 \mathrm{C} \delta 2$. Platinum coordination at His 15 has been reported to occur during the preparation of isomorphous derivatives'. The distances between the platinum sites and protein atoms suggested that some of the chloride ligands of hexachloroplatinate were replaced with protein atoms. The $\mathrm{N} \varepsilon 2$ and $\mathrm{N} \delta 1$ atoms of His 15 make hydrogen bonds with Ala $11 \mathrm{O}$ and Thr $89 \mathrm{O} \gamma 1$, respectively. Therefore, His 15 should undergo rotamer interconversion around its $C \beta-C \gamma$ bond in the soaked crystal.

The electron densities at sites 3, 4, and 5 slowly increased from $750 \mathrm{~s}$ and reached a plateau at $1.0-1.4$ electron $\AA^{-3}$. The complementary decrease (sites 1 and 2) and increase (sites 3, 4, and 5) of these electron densities indicated that (1) the binding rate constants of sites 1 and 2 are higher than those of sites 3,4 , and 5, and that (2) platinum ions at labile sites 1 and 2 moved to stable sites 3,4 , and 5 through binding equilibrium. The initial and subsequent processes 
Table 2 | Electron densities at platinum sites

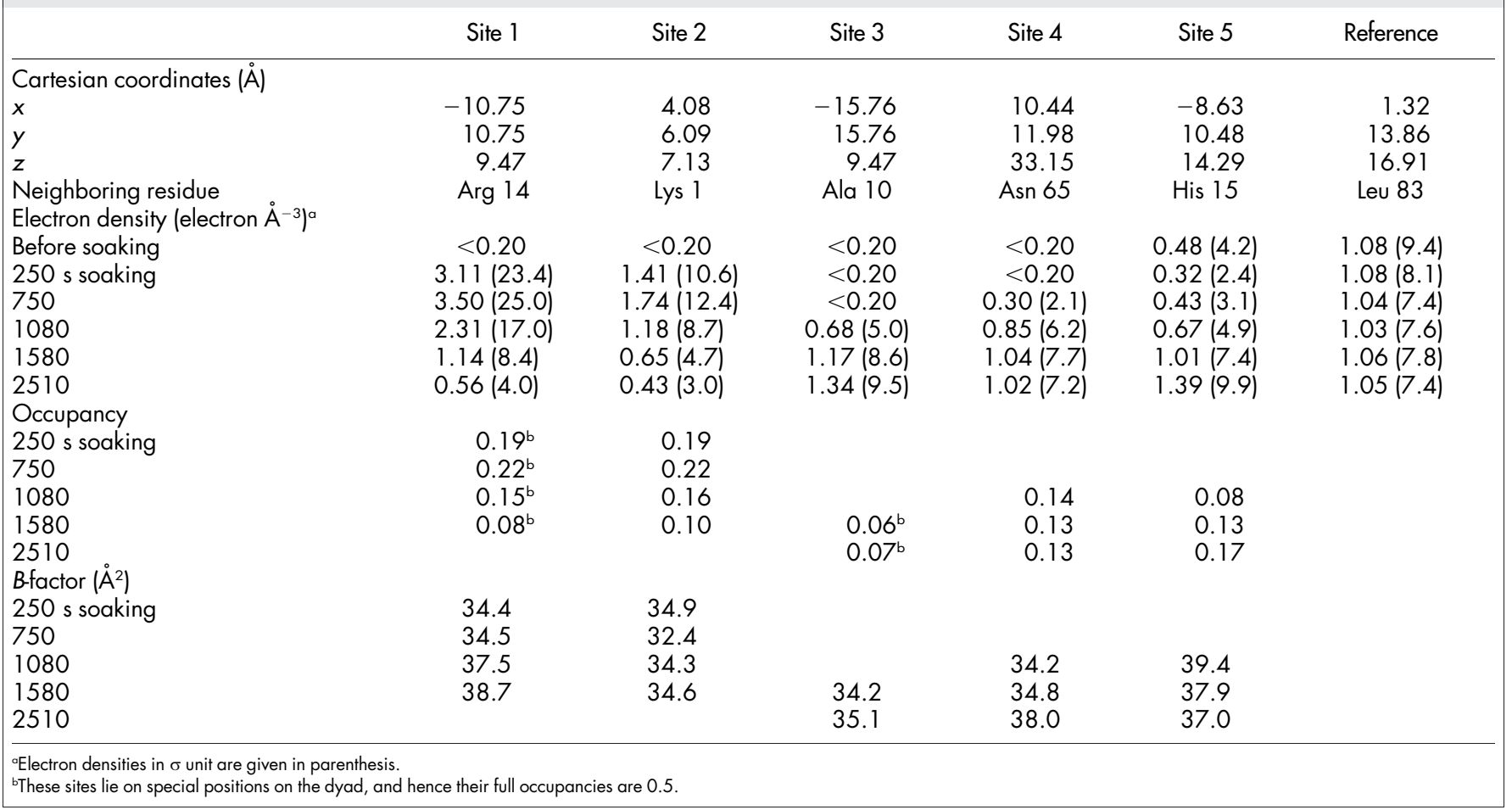

revealed from the LAC time dependence (Fig. 3) should correlate with these steps, though their kinetics also involve macroscopic processes such as platinum ion diffusion in the crystal. The reactions of platinum compounds and protein ${ }^{9,10}$ can be rather slow and complex. Any such reactions involving proteins in crystal should proceed in a similar manner.

The average LACs determined from the microtomographic analysis are equivalent to the total platinum content of 1.1 ions per asymmetric unit at $770 \mathrm{~s}$ and 1.6 ions at $2490 \mathrm{~s}$. However, the sums of platinum site occupancies were 0.44 at $750 \mathrm{~s}$ and 0.37 at $2510 \mathrm{~s}$. The discrepancy was rather larger at $2510 \mathrm{~s}$. It has been reported that a $22 \mathrm{~h}$ platinum-ion soak resulted in weak diffraction and low electron-density peaks of platinum, while a $10 \mathrm{~min}$ soak gave high peaks ${ }^{5}$. The discrepancies between the X-ray attenuation coefficient and platinum occupancies indicated that a number of platinum ions that were not identified in the electron density map existed in the

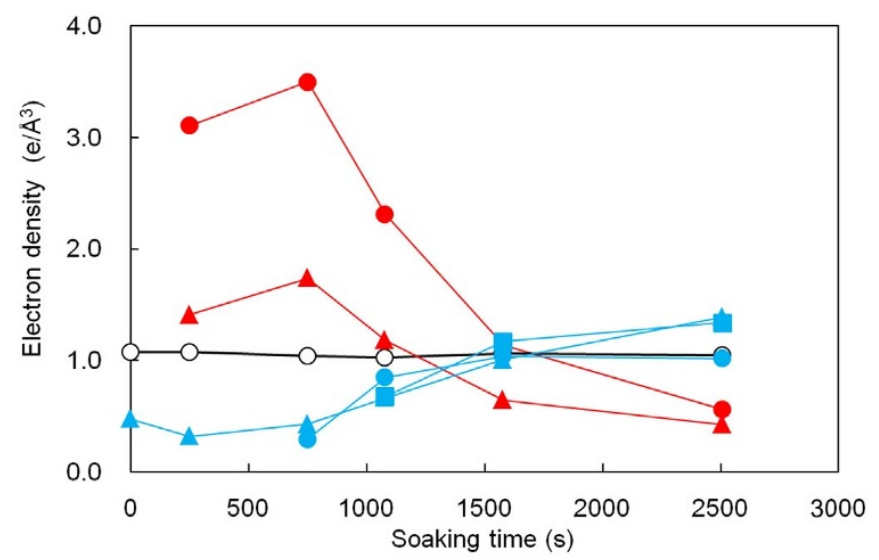

Figure $4 \mid$ Time dependence of electron densities at platinum sites. Site 1, $\bullet-\bullet$; site $2, \mathbf{\Lambda}-\mathbf{\Delta}$; site $3, \square-\square$; site $4, \bullet-\odot$; site $5, \mathbf{\Delta}-\mathbf{\Delta}$. Open circles $(\bigcirc-\bigcirc)$ represent densities at the position of water 180 of the starting model, which were almost constant throughout the soaking process. crystal and that the $B$-factors of platinum ions were higher than the values assumed in the refinement $\left(30-40 \AA^{2}\right)$.

\section{Discussion}

Crystallographic analysis of the soaked crystal indicated that the platinum coordination multifactorially develops over the course of $2500 \mathrm{~s}$. The microtomographic analysis indicated that the platinum distribution spread from the superficial layer to the crystal core within the same time scale. To explain these results, we propose a layered process of soaking (Fig. 2c). In this process, sites 1 and 2 correspond to the permeation front of platinum ions, and sites 3,4 , and 5 correspond to another layer succeeding the front. The initial permeation was observed in the cross section of the microtomographic image of the crystal. The change in the electron densities indicated that binding rates governed the coordination of sites 1 and 2 , as described above. The front then moved toward the crystal center during the soaking process but was simultaneously replaced by the succeeding layer in which platinum ions moved to sites 3,4 , and 5 . Finally, the front reached the crystal center and the succeeding layer dominated the entire crystal.

Although the soaking process of lysozyme crystal with a low solvent content of $41 \%$ gradually progressed over $2500 \mathrm{~s}$, it has been reported that diffusion into protein crystal with a high solvent content progresses within 10-15 $\mathrm{s}^{11}$. Therefore, a short soak in cryoprotectant solutions prior to cryo-crystallography should also cause spatial heterogeneity in crystal. The resultant heterogeneity can be captured by flash freezing. Recent progress in X-ray optics has allowed the application of micrometer X-ray beams to protein crystallography ${ }^{12}$. Radiation damage caused by synchrotron X-rays can be overcome by taking diffractions from fresh crystal regions even after diffractions have decayed with the absorbed dose. This can be performed by positional scanning of the flash-frozen crystal with an $\mathrm{X}$-ray microbeam ${ }^{13}$. However, possible structural heterogeneities owing to the short soak in the cryo-protectant solution can affect the atomic coordinates. We should consider heterogeneities that caused by crystal soaking might affect a dataset collected from multiple regions of the soaked crystal. 
Table 3 | Microtomographic data collection

X-ray energy (keV)

Pixel size $(\mu \mathrm{m})^{a}$

Pixel dynamic range (bits)

Viewing field size (pixels) ${ }^{a}$

No. of sample frames per dataset

Degrees per frame

Exposures per frame (ms)

Dataset collection time $(\mathrm{s})$

${ }^{a}$ Width $\times$ height.
$11.550,11.580$

$0.495 \times 0.495$

16

$2048 \times 2048$

450

0.40

200

120
The results obtained in this study suggest that the superficial and core regions of the crystal should be treated as having different structures. Consequently, a ligand-soaked crystal should be divided into regions containing initial and subsequent states of the proteinligand complex that vary in relation to their position in the crystal. The use of a microbeam is appropriate for exploiting such crystal heterogeneity. We propose that a pseudo time-resolved analysis can be performed by taking diffractions from the crystal edge and center if the ligand processing by the crystallized protein is slower than the binding rate.

Crystallographers have an implicit understanding that the crystal edge is different from the crystal core. The results obtained in this study illustrate this consensus quantitatively and suggest that the

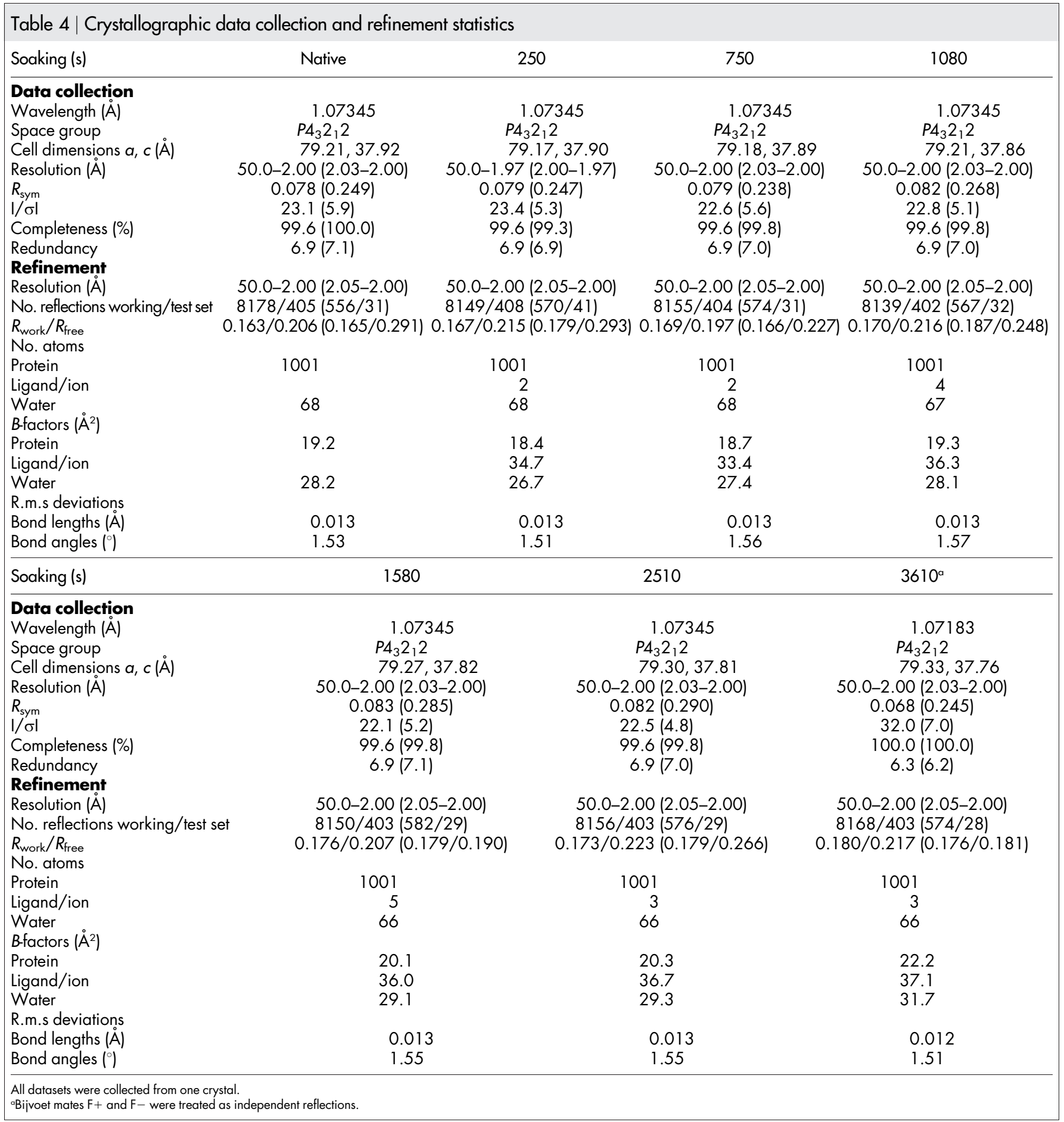


crystal structure itself can differ between the edge and core of the same crystal. Therefore, we should take into account the possibility that the composition of chemical and structural species resulting from the soaking process varies depending on the position from which diffractions were taken.

\section{Methods}

Crystallization. Chicken egg-white lysozyme (L6876, Sigma-Aldrich) was crystallized by the batch method. Lysozyme was dissolved in purified water at a concentration of $50 \mathrm{mg} / \mathrm{ml}$ and centrifuged at $20,000 \times g$ for $10 \mathrm{~min}$. The supernatant was mixed with an equal volume of $1.4 \mathrm{M} \mathrm{NaCl}$ aqueous solution containing $100 \mathrm{mM}$ sodium acetate ( $\mathrm{pH} 4.5$ ). Boro-silicate glass capillaries with an approximate diameter of $0.7 \mathrm{~mm}$ (W. Müller) were filled with the mixed solution and incubated at $20^{\circ} \mathrm{C}$ to grow lysozyme crystals on the capillary glass surface. Capillaries bearing crystals were connected to one end of a bulb tee connector (732-8302, BioRad) using brass tubes with outer/inner diameters of $1.2 / 0.8 \mathrm{~mm}$ and cemented using epoxy glue, as shown in Supplementary Fig. S3. Another end of the tee was attached to the goniometer head through a brass rod with a diameter of $1.2 \mathrm{~mm}$. The branch end of the tee was connected to a syringe using silicone rubber tubing. An aqueous solution containing $10 \mathrm{mM} \mathrm{K}_{2} \mathrm{PtCl}_{6}, 0.7 \mathrm{M} \mathrm{NaCl}$, and $50 \mathrm{mM}$ sodium acetate $(\mathrm{pH}$ 4.5 ) was introduced from this syringe upon data collection.

Microtomography. Simple-projection microtomographic analysis was performed at the BL20XU beamline ${ }^{14}$ of SPring-8. Tetragonal crystals with dimensions of $140-$ $150 \mu \mathrm{m}$ perpendicular to the $\left(\begin{array}{lll}1 & 1 & 0\end{array}\right)$ face and $100-150 \mu \mathrm{m}$ along the $c$ axis were used for microtomographic analysis. The sample capillary was mounted on the microtomograph by using a brass fitting specially designed for the brass pin sample. Absorption contrast radiographs were recorded at $300 \mathrm{~K}$ with a CMOS-based X-ray imaging detector (AA50 and ORCA-Flash4.0, Hamamatsu Photonics) using monochromatic radiation at $11.550 \mathrm{keV}$ and $11.580 \mathrm{keV}$. These X-ray energies correspond to energies below and above the $\mathrm{Pt} \mathrm{L}_{\mathrm{III}}$ edge, respectively. The X-ray energy was calibrated at the $\mathrm{Au} \mathrm{L}_{\mathrm{III}}$ edge $(11.9197 \mathrm{keV})$ by using gold foil. The X-ray flux density was moderated to be approximately $1.5 \times 10^{11}$ photons s $\mathrm{s}^{-1} \mathrm{~mm}^{-2}$ by adjusting the undulator gap. The detector was placed $20 \mathrm{~mm}$ from the capillary to make sufficient space for rotation of the samples with tubing. The viewing field and effective pixel size of the image detector are $1.013 \mathrm{~mm} \times 1.013 \mathrm{~mm}$ and $0.495 \mu \mathrm{m} \times$ $0.495 \mu \mathrm{m}$, respectively. It took $120 \mathrm{~s}$ to acquire each dataset. Datasets were obtained from the crystal before and after introducing the platinum solution into the capillary. Midpoints of the data acquisition were 135, 280, 770, 1090, 1590, and $2490 \mathrm{~s}$ after introduction. The data acquisition conditions are summarized in Table 3.

The obtained radiographs were subjected to a convolution-back-projection calculation using the program RecView ${ }^{15}$ (available from http://www.el.u-tokai.ac.jp/ ryuta/) accelerated with CUDA parallel-computing processors. Each microtomographic slice perpendicular to the sample rotation axis was reconstructed with this calculation. The spatial resolution of the reconstructed image was estimated to be $1.2 \mu \mathrm{m}$ by using three-dimensional square-wave patterns ${ }^{16}$. The crystal images were resliced to align the $c$ axis of the crystal lattice along the $x$ axis of the three-dimensional image, and the (1 110$)$ face parallel to the $x-y$ plane. Finally, voxels in each $10 \times 10 \times$ 10 bin corresponding to a volume of $4.95 \times 4.95 \times 4.95 \mu \mathrm{m}^{3}$ were averaged to improve the signal-to-noise ratio of the crystal images.

Crystallography. Diffraction intensities from crystals undergoing soaking were collected at the BL26B2 beamline ${ }^{17}$ of SPring- 8 . The incident X-ray profile was nearly circular with a full width at half maximum of $120 \mu \mathrm{m}$. The photon flux in this incident beam was attenuated to $8 \times 10^{9}$ photons $\mathrm{s}^{-1}$ by using $400-\mu \mathrm{m}$-thick aluminum. The $\mathrm{X}$-ray wavelength was calibrated at the $\mathrm{Au} \mathrm{L}_{\mathrm{III}}$ edge by using gold foil. Prior to the crystallographic data collection, an XAFS spectrum of the Pt $\mathrm{L}_{\text {III }}$ edge was acquired using a crystal soaked for $45 \mathrm{~min}$. The X-ray wavelength was set below the edge of this spectrum $(1.07345 \AA, 11.550 \mathrm{keV})$ to reduce the absorbed dose. Time series datasets were collected using a tetragonal crystal of $120-130 \mu \mathrm{m}$ in each dimension. Diffraction patterns of 45 frames were recorded at $300 \mathrm{~K}$ using a CCD detector (MarMosaic225, Rayonix) with an oscillation range of 2.0 degree per frame, an exposure time of $1.0 \mathrm{~s}$ per frame, and a crystal-to-detector distance of $150 \mathrm{~mm}$. Datasets were obtained before and after introducing the platinum solution into the capillary. It took $165 \mathrm{~s}$ to acquire each dataset. Midpoints of the data acquisition were $250,750,1080,1580$, and $2510 \mathrm{~s}$ from introduction. An additional dataset at $3610 \mathrm{~s}$ was collected at the peak $(1.07183 \AA, 11.568 \mathrm{keV})$ of the XAFS spectrum with an oscillation range of 1.0 degree per frame, an exposure time of $2.0 \mathrm{~s}$ per frame, and a total oscillation range of 150 degree. Diffraction images were processed with the HKL2000 program suite ${ }^{18}$. Statistics of the data collection are summarized in Table 4.

Native lysozyme structure $2 \mathrm{LYM}^{6}$ was used as a starting model for crystallographic refinement. Waters that exhibited electron densities lower than 0.5 electron $\AA^{-3}$ in the omit maps or that made contacts closer than $2.4 \AA$ were removed from the model. The crystallographic $R$-factor calculated using this model was 0.179 for the native dataset in the 50-2.0 $\mathrm{A}$ range. The model was subjected to crystallographic refinement using the program REFMAC ${ }^{19}$ of the CCP 4 suite $^{20}$. Statistics of the refined structure are summarized in Table 4.

Bijvoet difference maps were calculated using this native model and $750 \mathrm{~s}$ and $3610 \mathrm{~s}$ datasets. Five peaks higher than 0.15 electron $\AA^{-3}$ (corresponding to $5.7 \sigma$ in the $750 \mathrm{~s}$ map and $7.6 \sigma$ in the $3610 \mathrm{~s}$ map) were found in the Bijvoet difference maps and hence were identified as platinum sites. These sites also exhibited electron densities higher than 1.0 electron $\AA^{-3}$ in the omit maps calculated using the native model and derivative datasets, and they were tracked through the time series. The peak corresponding to water 180 of the starting model, which was removed owing to close contact with water 131, exhibited electron densities higher than 1.0 electron $\AA^{-3}$, though no Bijvoet peaks were found at this position. No other peaks met these electron density criteria.

Derivative models were generated by incorporating the platinum sites and subjected to crystallographic refinement using REFMAC. Since the average $B$-factor of waters in the native model was $28.2 \AA^{2}$, platinum occupancies were manually adjusted so that their $B$-factors fell within $30-40 \AA^{2}$. Then the platinum occupancies were further refined. Statistics of the refined structures are summarized in Table 4. Native (3WPJ), $250 \mathrm{~s}$ (3WU7), $750 \mathrm{~s}$ (3WPK), $1080 \mathrm{~s}$ (3WU8), $1580 \mathrm{~s}$ (3WU9), $2510 \mathrm{~s}$ (3WPL), and $3610 \mathrm{~s}$ (3WUA) models were submitted to the Protein Data Bank.

1. Velev, O. D., Kaler, E. W. \& Lenhoff, A. M. Surfactant diffusion into lysozyme crystal matrices investigated by quantitative fluorescence microscopy. J. Phys. Chem. B 104, 9267-9275 (2000).

2. Cvetkovic, A. et al. Quantifying anisotropic solute transport in protein crystals using 3-D laser scanning confocal microscopy visualization. Biotechnol Bioeng. 86, 389-398 (2004).

3. Geremia, S., Campagnolo, M., Demitri, N. \& Johnson, L. N. Simulation of diffusion time of small molecules in protein crystals. Structure 14, 393-400 (2006).

4. Helliwell, J. R. et al. Time-dependent analysis of $\mathrm{K}_{2} \mathrm{PtBr}_{6}$ binding to lysozyme studied by protein powder and single crystal X-ray analysis. Z. Kristallogr. 225, 570-575 (2010).

5. Joyce, M. G., Radaev, S. \& Sun, P. D. A rational approach to heavy-atom derivative screening. Acta Cryst. D 66, 358-365 (2010).

6. Kundrot, C. E. \& Richards, F. M. Collection and processing of X-ray diffraction data from protein crystals at high pressure. J. Appl. Cryst. 19, 208-213 (1986).

7. Henderson, R. Cryo-protection of protein crystals against radiation damage in electron and X-ray diffraction. Proc. R. Soc. Lond. B. 241, 6-8 (1990).

8. Williams, R. J., Dillin, D. R. \& Milligan, W. O. Structure refinement of potassium chloroplatinate by powder and single-crystal methods. Acta Cryst. B 29, 1369-1372 (1973).

9. Blake, C. C. F. The preparation of isomorphous derivatives. Adv. Protein Chem. 23, 59-120 (1968).

10. Blundell, T. L. \& Johnson, L. N. Preparation of heavy atom derivatives. In Protein Crystallography (Academic Press, London, 1976), pp. 183-239.

11. O'Hara, P., Goodwin, P. \& Stoddard, B. L. Direct measurement of diffusion rates in enzyme crystals by video absorbance spectroscopy. J. Appl. Cryst. 28, 829-834 (1995).

12. Ice, G. E., Budai, J. D. \& Pang, J. W. The race to $\mathrm{x}$-ray microbeam and nanobeam science. Science 334, 1234-1239 (2011).

13. Rasmussen, S. G. et al. Crystal structure of the human $\beta 2$ adrenergic G-proteincoupled receptor. Nature 450, 383-387 (2007).

14. Suzuki, Y. et al. Construction and commissioning of a $248 \mathrm{~m}$-long beamline with X-ray undulator light source. AIP Conf. Proc. 705, 344-347 (2004).

15. Mizutani, R. et al. Microtomographic analysis of neuronal circuits of human brain. Cereb. Cortex 20, 1739-1748 (2010).

16. Mizutani, R. et al. Submicrometer tomographic resolution examined using a micro-fabricated test object. Micron 41, 90-95 (2010).

17. Ueno, G. et al. RIKEN structural genomics beamlines at the SPring-8; high throughput protein crystallography with automated beamline operation. J. Struct. Funct. Genomics 7, 15-22 (2006).

18. Otwinowski, Z. \& Minor, W. Processing of X-ray Diffraction data collected in oscillation mode. Meth. Enzymol. 276, 307-326 (1997).

19. Murshudov, G. N., Vagin, A. A. \& Dodson, E. J. Refinement of macromolecular structures by the maximum-likelihood method. Acta Cryst. D 53, 240-255 (1997).

20. Winn, M. D. et al. Overview of the CCP4 suite and current developments. Acta Cryst. D 67, 235-242 (2011).

\section{Acknowledgments}

We thank Kiyoshi Hiraga (Technical Service Coordination Office, Tokai University) for assistance with the preparation of the brass fittings for the tomographic data collection. This work was supported in part by Grants-in-Aid for Scientific Research from the Japan Society for the Promotion of Science (nos. 25282250 and 25610126). The synchrotron radiation experiments were performed at SPring-8 with the approval of the Japan Synchrotron Radiation Research Institute (JASRI) (proposal nos. 2006B1014, 2012B0034, 2013A0034, 2013B0034, 2013B0041, and 2013B1889).

\section{Author contributions}

R.M. conceived and designed the study. R.M. and Y. Shimizu prepared the samples. R.M., Y. Shimizu, R.S., G.U., Y.N., A.T., K.U. and Y. Suzuki performed the synchrotron radiation experiments. R.M., Y. Shimizu, and R.S. analyzed the data. 


\section{Additional information}

Database Deposition: Native (3WPJ), 250 s (3WU7), 750 s (3WPK), 1080 s (3WU8), 1580 s (3WU9), 2510 s (3WPL), and 3610 s (3WUA) models were deposited in the Protein Data Bank database.

Supplementary information accompanies this paper at http://www.nature.com/ scientificreports

Competing financial interests: The authors declare no competing financial interests.

How to cite this article: Mizutani, R. et al. Spatiotemporal development of soaked protein crystal. Sci. Rep. 4, 5731; DOI:10.1038/srep05731 (2014).
This work is licensed under a Creative Commons Attribution-NonCommercialNoDerivs 4.0 International License. The images or other third party material in this article are included in the article's Creative Commons license, unless indicated otherwise in the credit line; if the material is not included under the Creative Commons license, users will need to obtain permission from the license holder in order to reproduce the material. To view a copy of this license, visit http:// creativecommons.org/licenses/by-nc-nd/4.0/ 